\title{
A Global Variational Filter for Restoring Noised Images with Gamma Multiplicative Noise
}

\author{
Nacira Diffellah \\ Department of Electronics, University of Biskra, Algeria and \\ Department of Electronics, Bachir El Ibrahimi University, ETA \\ Laboratory, Bordj Bou Arreridj, Algeria \\ diffellahn@gmail.com \\ Foued Derraz \\ Telecommunications Laboratory, Technology Faculty, \\ Abou Bekr Belkaid University, \\ Tlemcen, Algeria \\ fdrz@gmail.com
}

\author{
Zine-Eddine Baarir \\ LESIA Laboratory, \\ Mohamed Khider University, \\ Biskra, Algeria \\ zinedinebaarir@gmail.com \\ Abdelmalik Taleb-Ahmed \\ IEMN DOAE UMR CNRS 8520, \\ Polytechnic University of Hauts-de-France, \\ Valenciennes, France \\ taleb@uphf.fr
}

\begin{abstract}
In this paper, we focus on a globally variational method to restore noisy images corrupted by multiplicative gamma noise. The problem is assumed as a regularization problem in total variation (TV) framework with data fitting term which is deduced by maximizing the a-posteriori probability density (MAP estimation). We need to evaluate the proximal operator of a data fitting term then we numerically adapt the Douglas-Rachford (DR) splitting method to solve the problem. Real images with different levels of noise were used. To validate the effectiveness of the proposed method, the proposed method was compared with other variational models. Our method shows effective noise suppression, excellent edge preservation. Measures of image quality such as PSNR (peak signal-to-noise ratio), VSNR (visual signal-to-noise ratio) and SSIM (structural similarity index) explain the proposed model's good performance.
\end{abstract}

Keywords-multiplicative gamma noise; restoration; regularization; data fitting; total variation; MAP estimation; proximal operator; PSNR; SSIM; VSNR

\section{INTRODUCTION}

Noise suppression is one of the most important tasks in image processing. Digital images are very often contaminated by various types of noise which degrade them during acquisition or transmission. Many filtering techniques can be applied to restore the images, the best ones should completely remove noise as much as possible and preserve edges. Image noise can be classified as additive or multiplicative: In the first category the observed image is the sum of the true image and the noise, where the noise has often zero-mean and is described by its standard deviation. Each pixel in the noisy image is the sum of the true pixel value and a random Gaussian distributed noise value. In order to reduce an additive noise with a Gaussian distribution without sacrificing image detail, many approaches are used: wavelets [1-3], stochastic approaches [4], principal component analysis-based approaches [3, 5], variational [6-9], and proximal approaches [9-14]. In the second category, addition becomes multiplication. The objective is to restore a degraded image to its original form. Speckle noise is a granular noise that inherently exists in and degrades the quality of images. This type of noise occurs in coherent imaging systems such as medical ultrasound imaging, synthetic aperture radar (SAR), sonar, laser imaging and microscope images. The multiplicative noise is not independent and its distribution is generally not Gaussian, the speckle noise appears chaotic and unordered, it is difficult to interpret observed data. Speckle denoising is a very rigorous problem compared with additive Gaussian noise. To remove speckle noise, various methods have been proposed, the most of them are based on variational models such as anisotropic diffusion [15-18]. Methods introduced in [19-20] concerned nonlocal means, and variational approaches. The first nonconvex model was proposed in [21], it is called RLO model, it is an optimization problem to find the restored image and set up an objective function with a total variation term in order to preserve significant edges in images under the constraints: the mean and the variance of the noise $\eta$ are assumed to be 1 and $\sigma^{2}$ respectively. To solve the problem the gradient projection method is used. The objective function is nonconvex and it is very expensive to solve for a global minimizer of the model. The second nonconvex model, called AA model, is given in [22]. Authors used the classical MAP estimator to create it. To solve the problem they employed a gradient method.

Both multiplicative noise models suffer from nonconvexity. To overcome this difficulty, authors in [23] proposed a third model called SO. They transformed the multiplication operation into a summation using the logarithm and the derivation of this functional is based on the maximum a posteriori (MAP). The model is strictly convex in order to solve uniqueness problems of solution. In [24], authors studied a fast TV minimization method for multiplicative noise removal. They modified total variation regularization in the 
objective function. In [25], authors proposed a new formulation of a nonlinear complex diffusion filter NCDF, a regularization factor dependent on data, and an adaptive process. A new strictly variational convex model based on a quadratic penalty function was proposed in [26], is based on the statistical properties of noise, and a primal-dual algorithm was proposed to obtain the denoised image. The main idea in [27] was to decouple the image variable and the noise variable in the convex proposed model. The model, based on the dual formula in [28], is solved by using the dual problem using alternating direction method of multipliers. The regularization term in [29] is the combination of the classical total variation regularizer and a nonconvex regularizer. Authors in [30] developed a fixed-point proximity algorithm for solving a variational model. A model that combines a total generalized variational filter with shearlet transform was proposed in [31], an efficient alternating direction method of multipliers (ADMM) was developed for finding a solution. Our approach proceeds by splitting, each non-smooth function is used individually so as to yield an easily implementable algorithm, and is involved via its proximity operator $[11,32,33]$. We do not directly evaluate the proximity operator of total energy, we propose the use of proximal splitting methods. The solution is found through sequences of computations involving the proximal operator of data fitting and the proximal operator of regularization energy separately. The main idea is to split into two sub-functions: regularization term is the total variation norm and the data fitting is the indicator function. Our approach is compared to five existing methods: The first method is extended from [22] with speckle noise replaced by gamma distribution noise. The second is based on the ROF model [23]. The others are adaptive complex diffusion [25], convex variational method [26], and convex optimization [27].

\section{Methodology}

In this section, we will address our proposed method which is motivated by the application of proximal algorithm for solving the convex optimization problem in the context of variational restoration method. Given an observed image $f$, we reconstruct the clean image $u$ assuming $f=u . \eta$, where $\eta$ denotes a vector valued Gamma distributed random variable with expected value $\mu=1$ and variance $\sigma^{2}$ :

$$
\eta=\Gamma\left(\sigma=\frac{1}{\sqrt{L}}, \mu=1\right)
$$

where $\sigma$ is the standard deviation and $L$ is the number of observations. The mean and the variance of Gamma distribution are given by (2) and (3) respectively:

$$
\begin{aligned}
& \int_{\Omega} \eta=\int_{\Omega} \frac{f}{u}=1 \\
& \int_{\Omega}(\eta-1)^{2}=\int_{\Omega}\left(\left(\frac{f}{u}\right)^{2}-1\right)=\sigma^{2}
\end{aligned}
$$

A. Variational Regularization Model of Image Restoration via MAP Estimator

In this subsection, we present a model able to restore images corrupted with Gamma multiplicative noise.

\section{1) Gamma Density}

The distribution of the noise $\eta$ takes the form of $p(\eta ; L)$ :

$$
p(\eta ; L)= \begin{cases}\eta^{L-1} \frac{L^{L} e^{-L \eta}}{\Gamma(L)} & \text { if } \eta>0 \\ 0 & \text { if } \eta \leq 0\end{cases}
$$

This density can be written as:

$$
p\left(\frac{f}{u} ; L\right)=\frac{L^{L}}{(u)^{L} \Gamma(L)} f^{L-1} \cdot e^{-L\left(\frac{f}{u}\right)}
$$

\section{2) Maximum a Posteriori Estimation}

The Bayes formula delivers the a posteriori probability density of $u$ for a given value $f$ :

$$
p(u / f)=\frac{p(f / u) \cdot p(u)}{p(f)}
$$

where $p(u / f)$ denotes the a-posteriori probability density, $p(f / u)$ means the probability of $f$ given $u, p(u)$ is the apriori probability density, and $p(f)$ is the marginal probability or a priori $f$. We note that the samples of the noise on each pixel are mutually independent and identically distributed with density $p(\eta ; L)$. The Bayesian approach has the advantage that it allows incorporating additional prior information on $u$ via the a-priori probability density $p(u)$ into the reconstruction process.

To determine an approximation to the given image, we use the maximum a posteriori probability (MAP) estimator which maximizes the likelihood and respectively minimizes the negative log-likelihood function:

$$
\begin{aligned}
& \hat{u} \in \underset{u}{\arg \max } p(u / f) \Leftrightarrow \\
& \hat{u} \in \underset{u}{\arg \min }\{-\log [p(f / u)]-\log [p(u)]\}
\end{aligned}
$$

Probability densities are depending on the model of the noise in the given data. The first term of (7) is a data fidelity term:

$$
\underset{u}{\arg \min }\{-\log [p(f / u)]\} \simeq \underset{u}{\arg \min }\left\{L\left(\frac{f}{u}+\log u\right)\right\}
$$

The second term of (7) is Gibbs a-priori density [22]:

$$
p(u)=\frac{1}{Z} e^{-\gamma \phi(u)} \simeq e^{-\gamma \phi(u)}
$$

where $Z$ is the stabilization term, and $\gamma$ is a positive parameter. Equation (7) can be rewritten as: 


$$
\max _{u}\left\{p\left(\frac{u}{f}\right)\right\}=\underset{u}{\arg \min }\left\{\left(\log u+\frac{f}{u}\right)+\underset{\lambda}{\frac{\gamma}{L}} J(u)\right\}
$$

In this paper, we use total variation (TV) functional as regularization energy especially those of $\ell_{1}$-type. The search of estimated image is based on the minimization of two functionals [22]:

$$
\hat{u} \in \underset{u}{\arg \min }\left\{\int\left(\log u+\frac{f}{u}\right)+\lambda T V(u)\right\}
$$

where $\lambda$ is the regularization parameter which measures the tradeoff between a good fit and a regularized solution. We remark that the regularization term is non-differentiable and the data term is strongly nonlinear.

\section{B. Proposed TV Processing of Images with Gamma Multiplicative Noise}

We propose a TV based model to restore an image corrupted by Gamma multiplicative noise. To solve the minimization problem with the TV regularization, we suggest a Douglas-Rachford splitting approach. We use proximal splitting methods to solve convex optimization problems minimizing the energy functional of the form:

$$
\hat{u} \in \underset{u}{\arg \min }\{\underbrace{\int\left(\log u+\frac{f}{u}\right)}_{E_{\text {data }}}+\lambda \underbrace{\int|\nabla u|}_{E_{\text {reg }}}\}
$$

We will express (12) as:

$$
\underset{u}{\arg \min }\left\{\|u\|_{T V}+\lambda\left\|\log u+\frac{f}{u}\right\|_{2}\right\}
$$

The two sub-functions are: $E_{\text {reg }}(u)=\|u\|_{T V}=\|u\|_{1}$ and $E_{\text {data }}(u)=\left\|\log u+\frac{f}{u}\right\|_{2}, u \in \underset{u}{\arg \min } R_{\text {reg }}(u)+E_{\text {data }}(u)$,

$$
\begin{aligned}
& E_{\text {reg }}(u): \mathbb{R}^{n} \rightarrow \mathbb{R} \cup\{+\infty\}, E_{\text {data }}(u): \mathbb{R}^{n} \rightarrow \mathbb{R} \cup\{+\infty\} \\
& \left(\text { ridom }_{\text {reg }}\right) \cap\left(\text { ridom }_{\text {data }}\right) \neq \varnothing .
\end{aligned}
$$

Both functions $E_{\text {reg }}(u)$ and $E_{\text {data }}(u)$ are lower semicontinuous convex and not smooth on all points. The proximal operator of each one is defined as follows:

$$
\begin{aligned}
& \operatorname{Pr} o x_{E_{\text {reg }}} u: \mathbb{R}^{n} \rightarrow \mathbb{R}^{n} \\
& \operatorname{Pr} o x_{E_{\text {reg }}} u=\min _{y \in \mathbb{R}^{n}} E_{\text {reg }}(y)+\frac{1}{2}\|u-y\|^{2} \\
& \operatorname{Pr} o x_{E_{\text {data }}} u: \mathbb{R}^{n} \rightarrow \mathbb{R}^{n} \\
& \operatorname{Pr} o x_{E_{\text {reg }}} u=\min _{y \in \mathbb{R}^{n}} E_{\text {data }}(y)+\frac{1}{2}\|u-y\|^{2}
\end{aligned}
$$

1) Research of ProxEreg
$E_{\text {reg }} u$ is a non-differentiable convex function, we search the proximal $\operatorname{Pr} o x_{E_{r g}} u$ :

$$
\operatorname{Pr}_{\partial x_{\text {reg }}} x=\max \left(0,1-\frac{\gamma}{|x|}\right) x
$$

\section{2) Research of ProxEdata}

The novelty introduced in this paper consists of exploiting ProxEdata. First we prove the existence and uniqueness of ProxEdata then we get its appropriate equation. To reach this purpose and for more explanation, we propose the following demonstrations: $\nabla E_{\text {data }}=\frac{u-f}{u^{2}}$ and $\nabla^{2} E_{\text {data }}=\frac{2 f-u}{u^{3}}$. If $0<u<2 f$, we can prove that $E_{\text {data }}$ is strictly convex. The minimizer is unique $u=f$, the function $\log u+\frac{f}{u}$ has a minimum equal to $1+\log f$. We prove the existence of ProxEdata: From Example 4.10 in [10]:

$$
\phi(\xi)=\left\{\begin{array}{cc}
-\kappa \ln (\xi)+w \xi, \quad \varepsilon>0 \\
+\infty & \text { elsewhere }
\end{array}\right.
$$

then:

$$
\operatorname{Pr}_{\phi}(\xi)=\frac{\xi-w+\sqrt{|\xi-w|^{2}+4 \kappa}}{2}
$$

The term $E_{\text {data }}(u)$ :

$$
E_{\text {data }}(u)=\log (u)+\frac{f}{u}
$$

We rewrite (18):

$$
\phi(\xi)=\kappa \ln \left(\frac{1}{\xi}\right)+w \xi, \quad \xi>0
$$

Take $u=\frac{1}{\xi}$ then $\xi=\frac{1}{u},(21)$ is replaced by (22):

$\phi(u)=\kappa \ln (u)+w \frac{1}{u}, \quad \xi>0$

If $\kappa=1$ and $w=f$, we have:

$\phi(u)=\left\{\begin{array}{lr}\ln (u)+\frac{f}{u}, & u>0 \\ +\infty & \text { elsewhere }\end{array}\right.$

The proximity operator of $E_{\text {data }}$ is defined by:

$\operatorname{Prox}_{E_{\text {data }}}(u)=\frac{\frac{1}{u}-f+\sqrt{\left|\frac{1}{u}-w\right|^{2}+4}}{2}$ 
To solve our problem, we propose to proceed accordingly with the following algorithm 1:

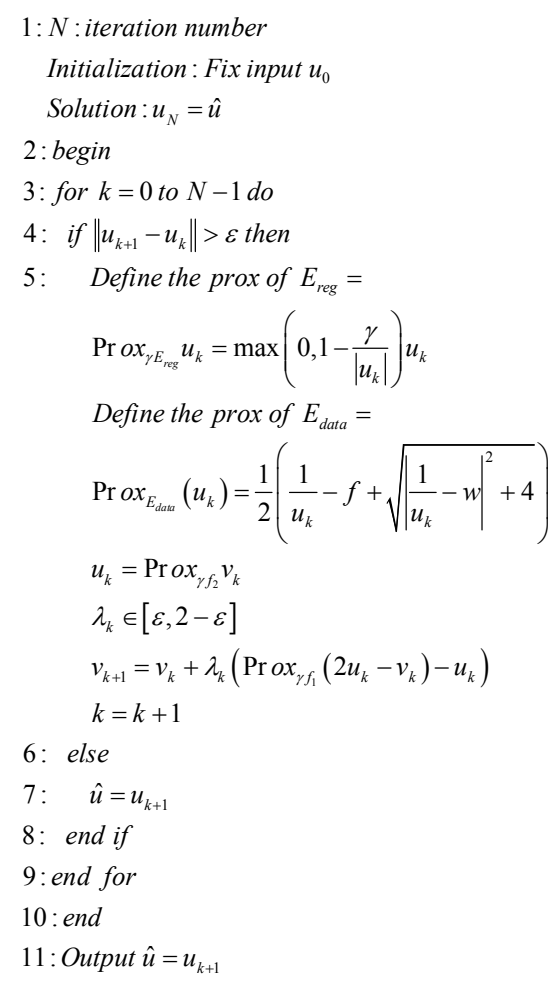

\section{RESULTS AND DISCUSSION}

This section presents the simulation results illustrating the performance of the proposed method. All the simulations were conducted in MATLAB and UNLocBoX-Matlab convex optimization toolbox.

\section{A. Denoising Performance}

Denoising performance is evaluated using:

- PSNR: Determines the degradation in the embedded image with respect to the original image. PSNR is more consistent in the presence of noise compared to SNR. The main advantages of PSNR are that it is very fast and easy to implement. The value of PSNR is larger, indicating that denoising effect is better.

- VSNR in $d B$ [34]. VSNR quantifies the visual fidelity of images.

- SSIM [35]. Compares two images using information about luminous, contrast and structure. SIM is a decimal value between $[-1,1]$.

We compare the original image $u$ with the restored version of image $\hat{u}$. The two images should have the same size. Equations (22)-(25) give the expressions of the quality measures MSE (mean squared error), PSNR (db), VSNR (db), and SSIM respectively:

$$
M S E=\frac{1}{M N} \sum_{i=0}^{M-1} \sum_{j=0}^{N-1}[u(i, j)-\hat{u}(i, j)]^{2}
$$

$$
\begin{gathered}
P S N R=10 \log _{10}\left(\frac{255^{2}}{M S E}\right) \\
V S N R=10 \log _{10}\left(\frac{C(u)}{V D^{2}}\right)
\end{gathered}
$$

where $C(u)$ denotes the root-mean-squared (RMS) contrast of the original image $u$ and $V D$ is visual distortion.

$$
\operatorname{SSIM}(u, \hat{u})=\frac{\left(2 \mu_{u} \mu_{\hat{u}}+C_{1}\right)\left(2 \sigma_{u \hat{u}}+C_{2}\right)}{\left(\mu_{u}^{2}+\mu_{\hat{u}}^{2}+C_{1}\right)\left(\sigma_{u}^{2}+\sigma_{\hat{u}}^{2}+C_{2}\right)}
$$

where $\operatorname{SSIM}(u, \hat{u})$ is the structural SIMilarity index between the two images, $\mu_{u}, \mu_{\hat{u}}$ is the average of $u, \hat{u}$ respectively, $\sigma_{u}, \sigma_{\hat{u}}$ is the standard deviation (the square root of variance) of $u, \hat{u}, \sigma_{u \hat{u}}$ is the standard deviation between $u$ and $\hat{u}$, and $C_{1}, C_{2}$ are positive constants.

\section{B. Result}

We compared our proposed algorithm to five already above mentioned methods: SRAD [15], ROF [23], adaptative method [25], convex [26], and convex mult method [27]. Experiments were performed on a parrot image of size $256 \times 256$ pixels, a cameraman of size $256 \times 256$ pixels, a cap image of size $512 \times 718$ pixels and a subway image of size $310 \times 450$ pixels (Figure 1). The digital images were contaminated by multiplicative noise with Gamma law of mean one and the number $L$ was $L=1, L=4$ and $L=10$.

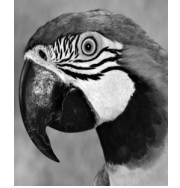

(a)

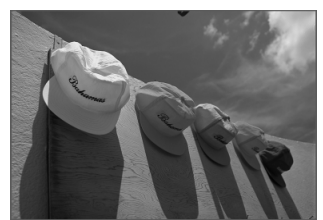

(c)

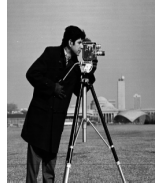

(b)

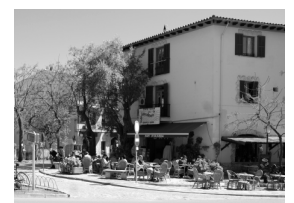

(d)
Fig. 1. Test images (a) Parrot, (b) Cameraman, (c) Cap, (d) Subway

In Figures 2-5, the denoised images are displayed. If the level noise is very high ( $L=1)$, the reconstructed image looks a little different to the original image since few texture and some edges and details have disappeared. For the other values of $L$, we can see that our (network) proposed model can effectively remove the speckle noise. It is very clear from Figure 6 that the restoration results of our model are visually better than the other methods' for all noise levels. The quantitative results of all methods are listed in Table I. 

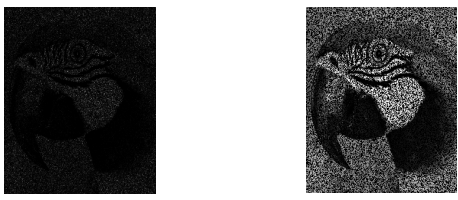

(a)

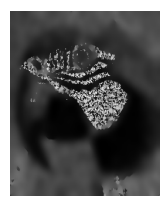

(b)

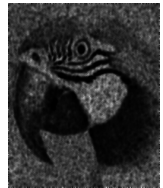

(c)

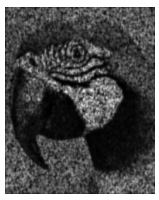

(d)

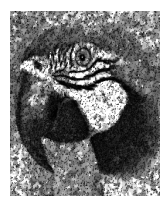

(e)

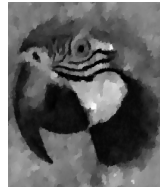

(f)

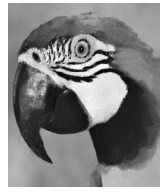

(g) (a')

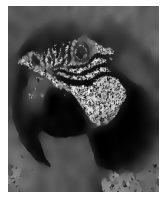

(b')

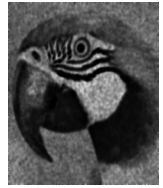

(c')

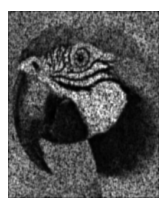

(d')

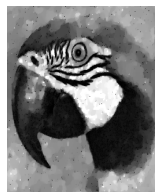

$\left(e^{\prime}\right)$

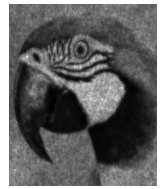

(f')

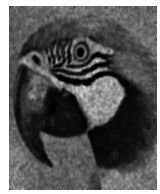

( $\left.\mathrm{g}^{\prime}\right)$

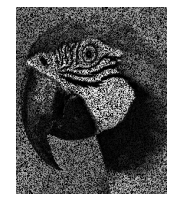

$\left(a^{\prime \prime}\right)$

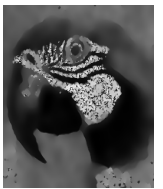

(b')

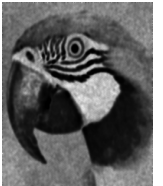

(c')

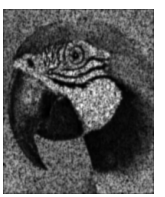

(d")

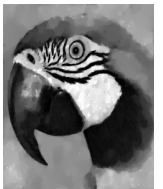

$\left(e^{\prime \prime}\right)$

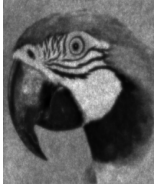

$\left(f^{\prime \prime}\right)$

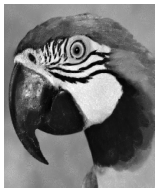

( $\left.\mathrm{g}^{\prime \prime}\right)$

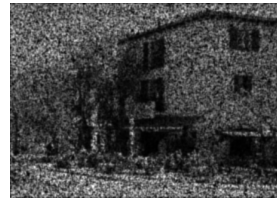

(a)

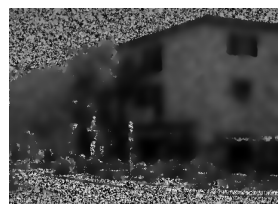

(b)

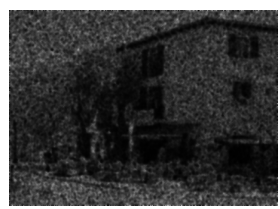

(c)

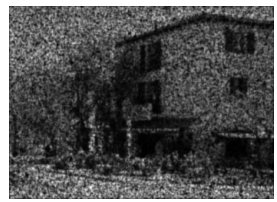

(d)

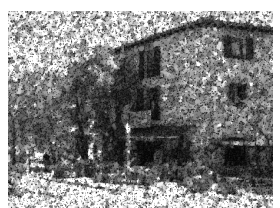

(e)

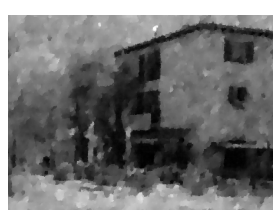

(f)

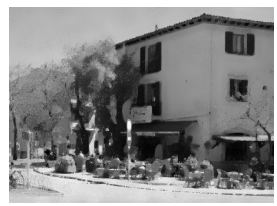

(g)

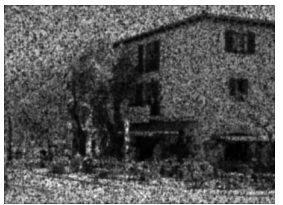

(a')

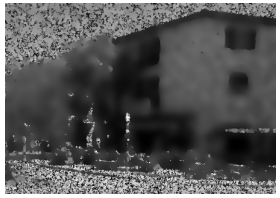

(b')

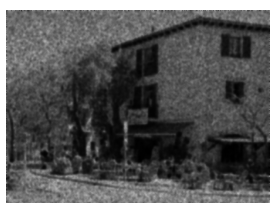

(c')

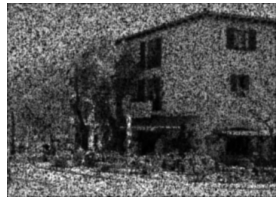

(d')

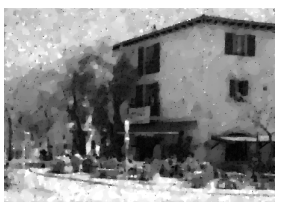

(e')

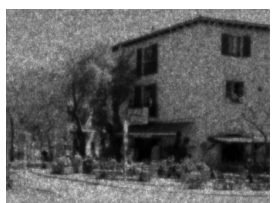

(f')

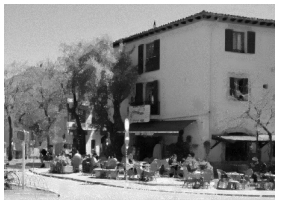

(g')

Fig. 2. Parrot: (a) degraded with Gamma noise $L=1$. Restored with (b) SRAD, (c) ROF, (d) Adaptative, (e) Covex, (f) Convex mult, (g) Proposed. (a') degraded with Gamma noise $L=4$. Restored with (b') SRAD, (c') ROF, (d') Adaptative, (e') Covex, (f') Convex mult, (g') Proposed. (a') Degraded with Gamma noise $L=10$. Restored with ( $\left.\mathrm{b}^{\prime \prime}\right)$ SRAD, (c') ROF, (d") Adaptative, ( $\left.\mathrm{e}^{\prime \prime}\right)$ Covex, (f) Convex mult, and ( $\left.\mathrm{g}^{\prime \prime}\right)$ Proposed

Fig. 3. Subway: (a) Degraded with Gamma noise $L=1$. Restored with (b) SRAD, (c) ROF, (d) Adaptative, (e) Covex, (f) Convex mult, (g) proposed. (a') Degraded with Gamma noise $L=4$. Restored with (b') SRAD, (c') ROF,

(d') Adaptative, (e') Covex, (f) Convex mult, and ( $\left.g^{\prime}\right)$ proposed. 


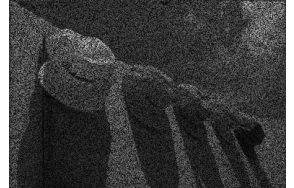

(a)

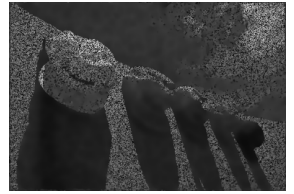

(b)

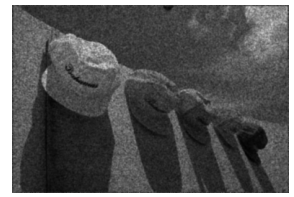

(c)

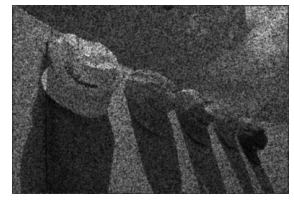

(d)

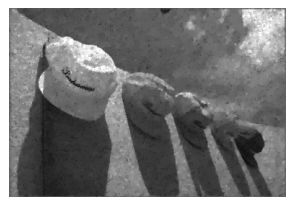

(e)

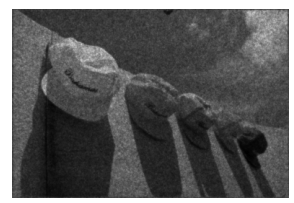

(f)

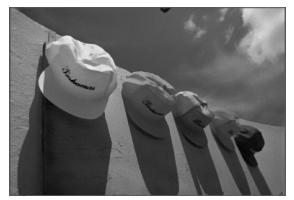

(g)

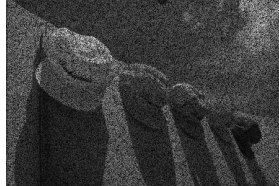

$\left(a^{\prime}\right)$

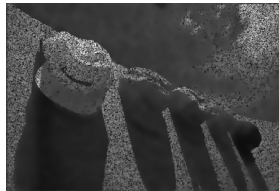

(b')

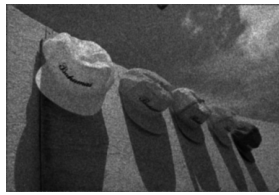

(c')

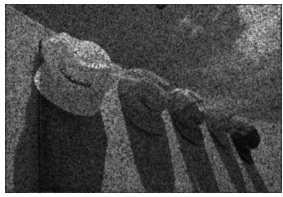

$\left(d^{\prime}\right)$

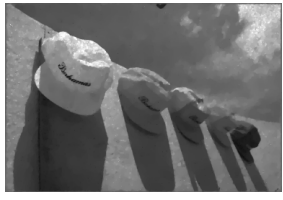

$\left(\mathrm{e}^{\prime}\right)$

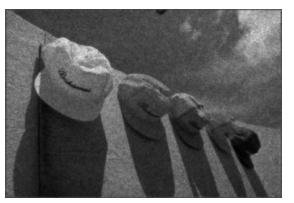

$\left(f^{\prime}\right)$

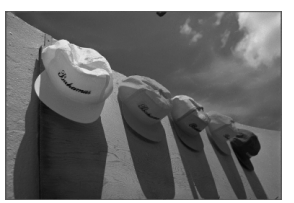

$\left(\mathrm{g}^{\prime}\right)$
Fig. 4. Cap: (a) degraded with Gamma noise $L=4$. Restored with (b) SRAD, (c) ROF, (d) Adaptative, (e) Covex, (f) Convex mult, (g') proposed (a') Degraded with Gamma noise $L=10$. Restored with (b') SRAD, (c') ROF, (d') Adaptative, (e') Covex, (f') Convex mult, and ( $\left.\mathrm{g}^{\prime}\right)$ Proposed.

Table I presents the PSNR, SSSIM and VSNR values for the test images of all methods at various noise levels. After inspection, it is evident that the proposed method provides higher PSNR, SSIM and VSNR for all numbers of look compared to other techniques. The proposed technique gives the best quality for all the numbers of look.

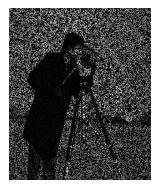

(a)

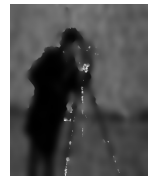

(b)

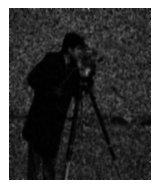

(c)

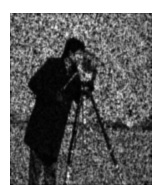

(d)

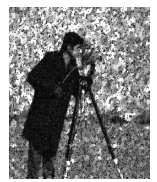

(e)

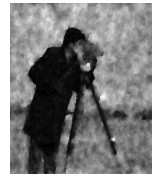

(f)

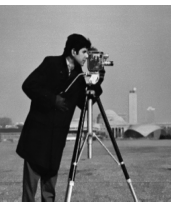

(g)

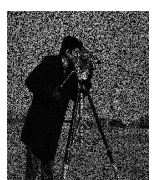

(a')

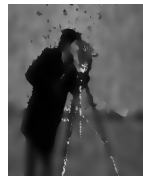

(b')

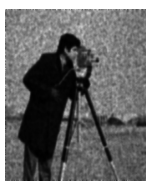

(c')

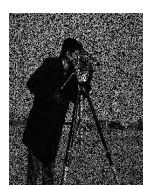

(d')

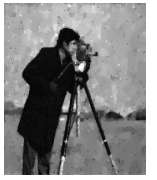

$\left(\mathrm{e}^{\prime}\right)$

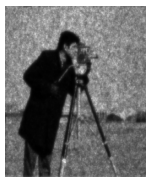

(f')

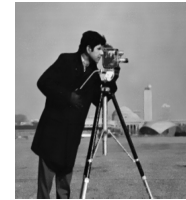

$\left(\mathrm{g}^{\prime}\right)$

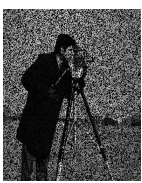

$\left(a^{\prime \prime}\right)$

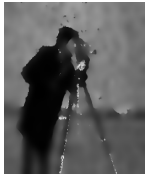

$\left(b^{\prime \prime}\right)$

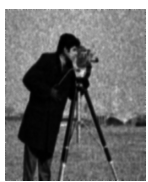

(c'

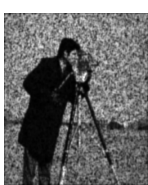

(d')

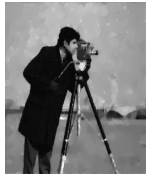

$\left(\mathrm{e}^{\prime \prime}\right)$

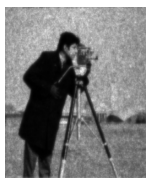

$\left(f^{\prime \prime}\right)$

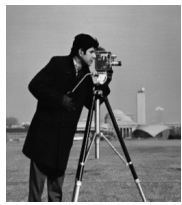

( $\left.g^{\prime \prime}\right)$
Fig. 5. Cameraman. (a) Degraded with Gamma noise $L=1$. Restored with (b) SRAD, (c) ROF, (d) Adaptative, (e) Covex, (f) Convex mult, (g) Proposed. (a') Degraded with Gamma noise $L=4$. Restored with (b') SRAD, (c') ROF, (d') Adaptative, (e') Covex, (f') Convex mult, (g') Proposed. (a') Degraded with Gamma noise $L=10$. Restored with (b') SRAD, (c') ROF, $\left(d^{\prime \prime}\right)$ Adaptative, (e') Covex, (f) Convex mult, and ( $\left.g^{\prime}\right)$ Proposed. 
TABLE I. QUANTITY RESULTS

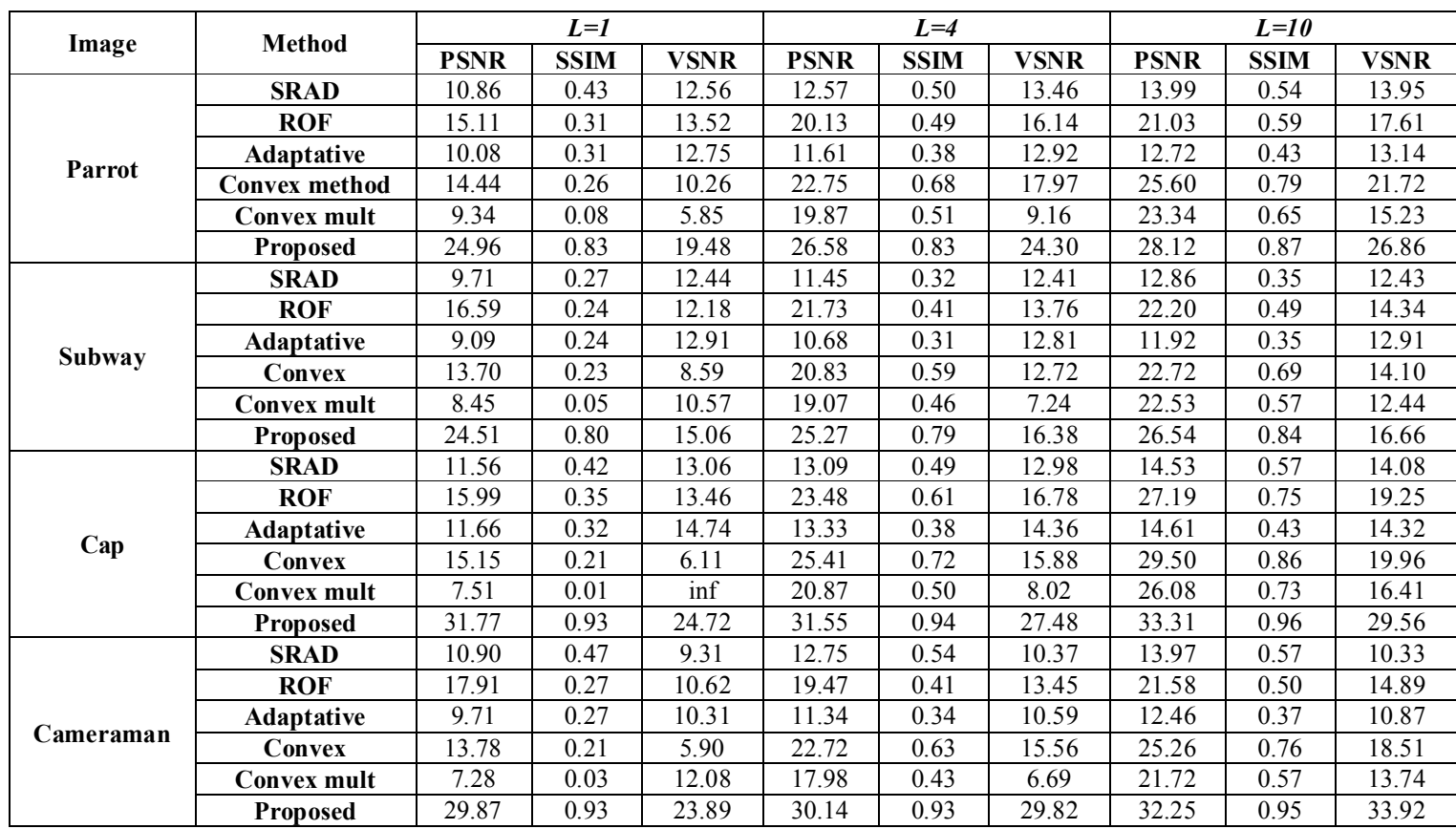

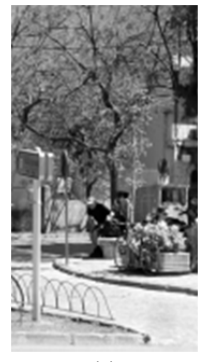

(a)

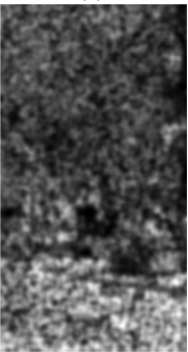

(e)

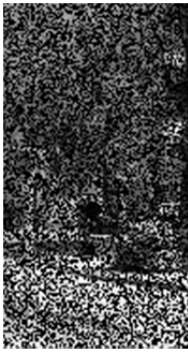

(b)

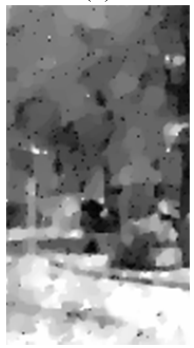

(f)

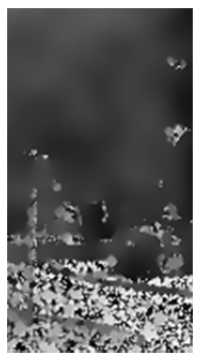

(c)

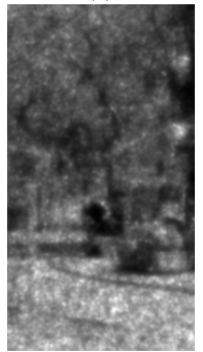

(g)

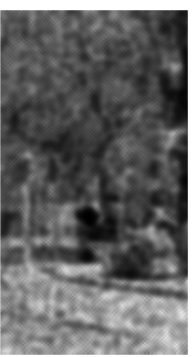

(d)

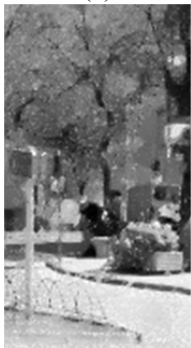

(h)

Fig. 6. Part of subway image. (a) Original image, (b) Degraded with Gamma noise $L=4$. Restored with (c) SRAD, (d) ROF, (e) Adaptative, (f) Convex, (g) Convex mult, and (h) Proposed.

\section{CONCLUSION}

In this paper, variational methods have been proposed to handle the restoration problem under the Gamma distributed multiplicative noise (with mean one and deviation $\frac{1}{\sqrt{L}}$ ). The proposed model is strictly convex if noisy image $f>0$ and original image $0<u<2 f$. The proposed model consists of a data fitting term, and a total variation regularizer. Image $T V$ is utilized as the regularization term in order to preserve significant edges in images. To solve the restoration problem, the Douglas-Rachford splitting algorithm was used. For finding the solution of this algorithm, we described and searched the proximity operators of two terms. We have proposed a new proximal data fitting for the solution of optimization problem from the minimization of energy functional. Numerical experiments demonstrate that the proposed model has a good performance and produces the best values of PSNR, SSIM and a good value of VSNR. The proposed approach substantially reduces the "staircase" and "blurring" effect, preserves more details and suppress Gamma multiplicative noise successfully. 
This paper opens a new door to perspectives, future work will include variational method using the proposed approach to restore the noised and blurred images corrupted by both multiplicative and additive noise. Moreover, we can exploit this idea for the denoising of image sequences [37].

\section{REFERENCES}

[1] D. L. Donoho, I. M. Johnstone, "Adapting to unknown smoothness via wavelet shrinkage", Journal of the American Statistical Association, Vol. 90, No. 432, pp. 1200-1224, 1995

[2] P. Bao, M. Zhang, "Noise reduction for magnetic resonance images via adaptive multiscale products thresholding", IEEE Transactions on Medical Imaging, Vol. 22, No. 9, pp. 1089-1099, 2003

[3] L. Zhang, R. Zhang, X. Wu, D. Zhang, "Pca-based spatially adaptive denoising of cfa images for single-sensor digital cameras", IEEE Transactions on Image Processing, Vol. 18, No. 4, pp. 797-812, 2009

[4] S. Geman, D. Geman, "Stochastic relaxation, gibbs distributions, and the bayesian restoration of images", IEEE Transactions on Pattern Analysis and Machine Intelligence, Vol. 6, No. 6, pp. 721-741, 1984

[5] L. Zhang, W. Donga, D. Zhang, G. Shib, "Two-stage image denoising by principal component analysis with local pixel grouping", Pattern Recognition, Vol. 43, No. 4, pp. 1531-1549, 2010

[6] L. I. Rudin, S. Osher, E. Fatemi, "Nonlinear total variation based noise removal algorithms", Physica D: Nonlinear Phenomena, Vol. 60, No. 14, pp. 259-268, 1992

[7] G. Aubert, P. Kornprobst, Mathematical Problems in Image Pro-cessing Partial Differential Equations and the Calculus of Variations, Springer, 2006

[8] A. Chambolle, "An algorithm for total variation minimization and applications", Journal of Mathematical Imaging and Vision, Vol. 20, No. 1-2, pp. 89-97, 2004

[9] P. L. Combettes, "Solving monotone inclusions via compositions of non expansive averaged operators", Optimization: A Journal of Mathematical Programming and Operations, Vol. 53, No. 5-6, pp. 475504,2004

[10] C. Chaux, P. L. Combettes, J. C. Pesquet, V. R. Wajs, “A variational formulation for frame-based inverse problems", Inverse Problems, Vol. 23, No. 1, pp. 1495-1518, 2007

[11] P. L. Combettes, J. C. Pesquet, "A douglas-rachford splitting ap-proach to nonsmooth convex variational”, IEEE Journal of Selected Topics in Signal Processing, Vol. 1, No. 4, pp. 564-574, 2007.

[12] P. L. Combettes, J. C. Pesquet, "A proximal decomposition method for solving convex variational inverse problems", Inverse Problems, Vol. 24, No. 6, pp. 065014, 2008

[13] J. Huang, S. Zhang, H. Li, D. Metaxas, "Composite splitting algorithms for convex optimization", Computer Vision and Image Understanding, Vol. 115, No. 12, pp. 1610-1622, 2011

[14] Q. Fan, D. Jiang, Y. Jiao, “A multi-parameter regularization model for image restoration", Signal Processing, Vol. 114, pp. 131-142, 2015

[15] Y. Yu, S. Acton, "Speckle reducing anisotropic diffusion", IEEE Transactions on Image Processing, Vol. 11, pp. 1260-1270, 2002

[16] S. A. Fernandez, C. A. Lopez, "On the estimation of the coefficient of variation for anisotropic diffusion speckle filtering", IEEE Transactions on Image Processing, Vol. 15, No. 11, pp. 2694-2701, 2006

[17] K. Krissian, C. Westin, R. Kikinis, K. Vosburgh, "Oriented speckle reducing anisotropic diffusion", IEEE Transactions on Image Processing, Vol. 16, No. 5, pp. 1412-1424, 2007

[18] G. Liu, X. Zeng, F. Tian, Z. Li, K. Chaibou, "Speckle reduction by adaptive window anisotropic diffusion”, Signal Processing, Vol. 89, No. 11, pp. 2233-2243, 2009

[19] A. Buades, B. Coll, J. M. Morel, "A review of image denoising algorithms, with a new one", SIAM Journal on Multiscale Modeling and Simulation, Vol. 4, No. 2, pp. 490-530, 2005

[20] C. A. Deledalle, L. Denis, F. Tupin, "Iterative weighted maximum likelihood denoising with probabilistic patchbased weights", IEEE Transactions on Image Processing, Vol. 18, No. 12, pp. 2661-2672, 2009
[21] L. Rudin, P. L. Lions, S. Osher, "Multiplicative denoising and deblurring: Theory and algorithms", in: Geometric Level Set Methods in Imaging, Vision, and Graphics, Springer, 2003

[22] G. Aubert J. F. Aujol, "A variational approach to removing multiplicative noise", SIAM Journal on Applied Mathematics, Vol. 68, No. 4, pp. 925-946, 2008

[23] J. Shi, S. Osher, "A nonlinear inverse scale space method for a convex multiplicative noise model", SIAM Journal on Imaging Sciences, Vol. 1, No. 4, pp. 294-321, 2008

[24] Y. M. Huang, M. K. Ng, Y. W. Wen, "A new total variation method for multiplicative noise removal", SIAM Journal on Imaging Sciences, Vol. 2, No. 1, pp. 20-40, 2009.

[25] R. Bernardes, C. Maduro, P. Serranho, A. Arajo, S. Barbeiro, J. CunhaVaz, "Improved adaptive complex diffusion despeckling filter", Optics Express, Vol. 18, No. 23, pp. 24 048-24 059, 2010

[26] Y. Dong, T. Zeng, "A convex variational model for restoring blurred images with multtiplicative noise", SIAM Journal on Imaging Sciences, Vol. 6, No. 3, pp. 1598-1625, 2013

[27] X. L. Zhao, F. Wang, M. K. Ng, “A new convex optimization model for multiplicative noise and blur removal", SIAM Journal on Imaging Sciences, Vol. 7, No. 1, pp. 456-475, 2014

[28] Y. Han, C. Xu, G. Baciu, X. Feng, "Multiplicative noise removal combining a total variation regularizer and a nonconvex regularizer", International Journal of Computer Mathematics, Vol. 91, No. 10, pp. 2243-2259, 2014

[29] Y. Hao, J. Xu, "An effective dual method for multiplicative noise removal", Journal of Visual Communication and Image Representation, Vol. 25, No. 2, pp. 306-312, 2014

[30] Y. Han, C. Xu, G. Baciu, X. Feng, "Multiplicative noise removal combining a total variation regularizer and a nonconvex regularizer", International Journal of Computer Mathematics, Vol. 91, No. 10, pp. 2243-2259, 2014

[31] J. Lu, L. Shen, C. Xu, Y. Xu, "Multiplicative noise removal in imaging: An exp-model and its fixed-point proximity algorithm", Applied and Computational Harmonic Analysis, Vol. 41, No. 2, pp. 518-539, 2016

[32] A. Ullah, W. Chen, M. A. Khan, H. G. Sun, "A new variational approach for multiplicative noise and blur removal", PLoS One, Vol. 12, No. 1, pp. e0161787, 2017

[33] P. L. Combettes, V. R. Wajs, "Signal recovery by proximal forward backward splitting", SIAM Journal on Multiscale Modeling \& Simulation, Vol. 4, No. 4, pp. 1168-1200, 2005

[34] H. H. Bauschke, R. S. Burachik, P. L. Combettes, V. Elser, D. Rusel Luke, H. Wolkowicz, Fixed-Point Algorithms for Inverse Problems in Science and Engineering. Springer, 2011

[35] D. M. Chandler, S. S. Hemami, "Vsnr: A wavelet-based visual signal-tonoise ratio for natural images", IEEE Transactions on Image Processing, Vol. 16, No. 9, pp. 2284-2298, 2007

[36] Z. Wang, A. Bovik, H. Sheikh, E. Simoncelli, "Image quality assessment: From error visibility to structural similarity", IEEE Transactions on Image Processing, Vol. 13, No. 4, pp. 600-612, 2004

[37] M. V. Sarode, P. R. Deshmukhnn, "Image sequence denoising with motion estimation in color image sequences", Engineering, Technology \& Applied Science Research, Vol. 1, No. 6, pp. 139-143, 2011 